\title{
SUMMARY OF SPIN SESSION TALKS $\dagger$
}

\author{
H. Spinka
}

High Energy Physics Division

Argonne National Laboratory, Argonne, Illinois 60439

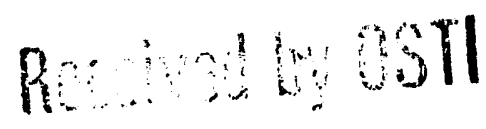

ONT 281991

\section{ABSTRACT}

Measurements of spin observables in scattering experiments have been useful to explore a wide variety of medium and high energy physics phenomena. Important new results were presented in each of the spin, combined hadron/spin and combined electrons and muons/spin sessions at this conference. Talks concerning spin effects in other sessions and in one of the hadron/spin parallel sessions are summarized by other speakers.

The spin talks summarized in this paper are divided into four topics: tests of symmetry principles, lepton scattering, hadron scattering experiments, and new developments in spin-related technologies.

\section{SYMMETRY TESTS}

W. Jacobs of Indiana University spoke about the charge symmetry test performed in a recently completed experiment involving a vertically polarized neutron beam scattering from a vertically polarized proton target. The neutron and proton analyzing powers $(A n(\theta)$ and $A p(\theta))$ were measured simultaneously and compared. The difference $\Delta A=A n-A p$, plotted in Fig. 1 , is expected to be zero if charge symmetry is valid. Combining the data over the c.m. angular range of $82-116^{\circ}$, where $\left.\angle A p(\theta)\right\rangle$ is expected to vanish, gives $\langle\Delta A\rangle=(33.1 \pm 5.9 \pm 4.3) \times 10^{-4}$ at a neutron beam kinetic energy of $183 \mathrm{MeV}$. These results were interpreted to indicate the importance of $\rho-\omega$ mixing contributions in meson exchange model calculations of this charge symmetry breaking effect.

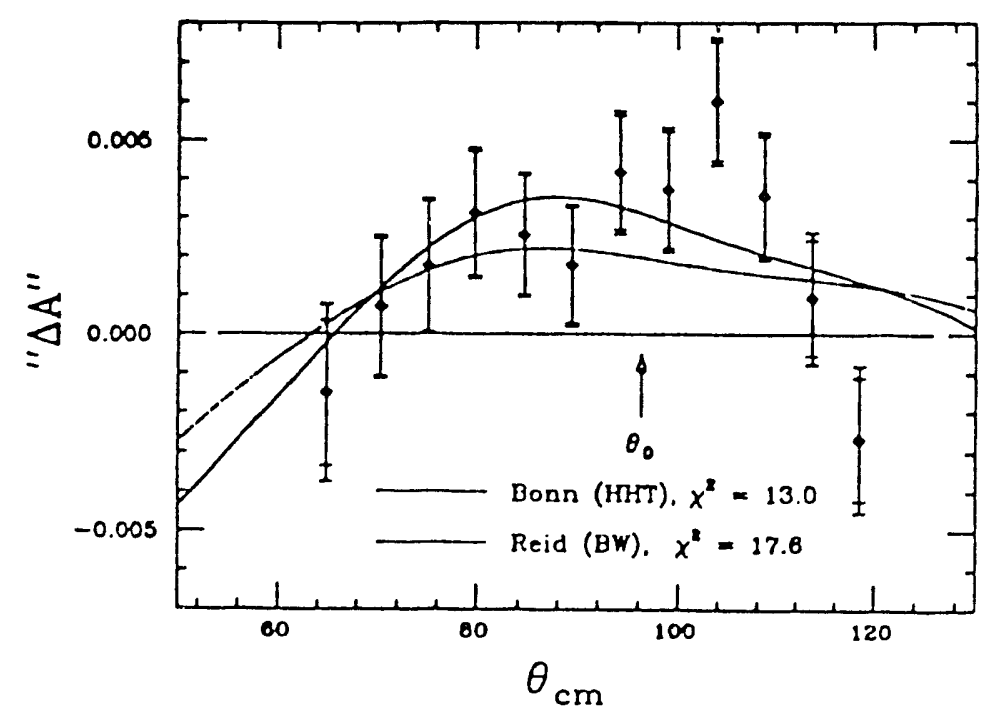

Fig. 1. The measured difference in analyzing powers for the charge symmetry test as a function of c.m. angle. 
J. Sromicki of ETH Zürich described a search for time reversal violation in the decay of polarized ${ }^{8} \mathrm{Li}$. The ${ }^{8} \mathrm{Li}$ nuclei were produced at PSI by the ${ }^{{ }^{2} \mathrm{Li}}+\overrightarrow{\mathrm{d}} \rightarrow$ ${ }^{8} \mathrm{~L} \overrightarrow{\mathrm{i}}+\mathrm{p}$ reaction using a $10 \mathrm{MeV}$ polarized deuteron beam. The transverse polarization of the $\beta$-decay electrons was measured and found to be consistent with zero, as expected from time reversal invariance. Expressing the results in terms of the ratio of anomalous weak tensor coupling constants to the axial vector coupling constant leads to the limits $-0.03 \leq \operatorname{Im}\left(\left[\mathrm{C}_{\mathrm{T}}+\mathrm{C}_{\mathrm{T}^{\prime}}\right] / \mathrm{C}_{\mathrm{A}}\right) \leq 0.05$.

J. Jacobs of the University of Washington deszribed a measurement of the permanent electric dipole moment in ${ }^{199} \mathrm{Hg}$. Time reversal invariance requires this moment to vanish. This nucleus was chosen since mercury is easily optically polarized and the expected effects would scale as $Z^{2}$. The preliminary limit for the electric dipole moment of $199 \mathrm{Hg}$ was reported to be $5 \times 10^{-27} \mathrm{e} \mathrm{cm}$. This value provides the most stringent upper limit for certain types of time reversal violating hadronic and semileptonic interactions. Additional runs are planned to reduce the limit.

N. Severijns of U.C.L. Louvain-la-Neuve described a search for the effects of possible right-handed currents in the $\beta$-decay of polarized ${ }^{107}$ In nuclei. The ratio of the longitudinal polarization of the positrons emitted antiparallel $\left(\mathrm{P}_{-}\right)$and parallel $\left(\mathrm{P}_{+}\right)$ to the ${ }^{07}$ In spin direction was measured to be $P_{-} / P_{+}=0.686 \pm 0.054$. The value expected from a pure V-A interaction is $0.771 \pm 0.017$. The data allow a $90 \%$ confidence level limit to the ratio of left- and right-handed $W$ masses of $\left[M\left(W_{L}\right) / M\left(W_{R}\right)\right]^{2}$ $<0.14$, or $\mathrm{M}\left(\mathrm{W}_{\mathrm{R}}\right)>215 \mathrm{GeV}$.

Parity violation (PV) in electron scattering experiments was discussed by $D$. Beck of the University of Illinois, M. Musolf of MIT, and R. Carlini of CEBAF. The PV effects originate from an interference of weak and electromagnetic terms describing the interaction. The results from the BATES ${ }^{12} \mathrm{C}\left(\overrightarrow{\mathrm{e}}, \mathrm{e}^{\prime}\right)$ measurement were presented, and a new ${ }^{1} \mathrm{He}\left(\overrightarrow{\mathrm{e}}, \mathrm{e}^{\prime}\right)$ experiment scheduled to run in 1993 at BATES was described. Future plans were also discussed to perform a higher precision PV measurement at CEBAF, by exploiting the outstanding beam position and emittance stability anticipated at the new accelerator and new technological improvements in polarized electron sources. In the past, these electron scattering PV experiments were hoped to provide precision tests of the Standard Model and searches for new physics. However, uncertainties in knowledge of the relevant hadronic physics have been identified that may cloud the interpretation of these measurements. Thus ( $\left.\vec{e}, e^{\prime}\right)$ PV experiments at medium energies will probably be more useful to study nuclear structure and nucleon strangeness and to determine nucleon form factors under the assumption that the Standard Model is valid.

\section{LEPTON SCATTERING}

J. Cameron of Indiana University and D. Beck described some of the electron scattering experiments involving spin at BATES. Spin significantly expands the capabilities of measurements for nucleon and nuclear structure, allowing the separation of effects from various processes contributing to electron scattering. As a consequence, a substantial fraction of the present BATES and planned CEBAF experimental programs will utilize some spin degree of freedom, either polarized beams or targets or recoil polarimeters to measure the spin of the outgoing nucleon.

Two experiments to measure the neutron elastic form factors $G_{E}^{n}$ and $G_{M}^{n}$ were discussed. The first studied the ${ }^{3} \mathrm{He}(\overrightarrow{\mathrm{e}}, \mathrm{e})$ reaction near $\mathrm{Q}^{2}=0.2 \mathrm{GeV}^{2}$ using both a spin exchange and a "metastability exchange" optically pumped polarized ${ }^{3} \mathrm{He}$ target. 
Reasonable agreement for the spin observables AT' and ATL' is obtained with the two targets and with theoretical predictions. The metastability exchange data for ATL' give $G_{E}^{n}=0.07 \pm 0.10 \pm 0.04$. The second experiment is presently collecting data and uses the reaction near $180^{\circ}$ to obtain $\mathrm{G}_{\mathrm{E}}^{\mathrm{n}}$ more directly. This reaction and kinematics were chesen in order to be less sensitive to final state interactions, meson exchange currents, and the deuteron wave function.

An electric quadrupole transition strength can arise in electroproduction experiments if the $\mathrm{N}-\Delta$ transition is not a purely quark spin flip process. Measurements of the ${ }^{1} \mathrm{H}\left(\overrightarrow{\mathrm{e}}, \mathrm{e}^{\prime} \mathrm{p}\right)$ reaction, with the proton out of the scattering plane (see Fig. 2), and the ${ }^{1} \mathrm{H}\left(\overrightarrow{\mathrm{e}}, \mathrm{e}^{\prime} \mathrm{p}\right)$ reaction, with a carbon polarimeter to obtain the outgoing proton spin, were described. Both experiments are sensitive to the ratios of charge and electric quadrupole to dipole transition amplitudes, C2/M1 and E2/M1, and both have minimal effects from nonresonant processes and final state interactions.
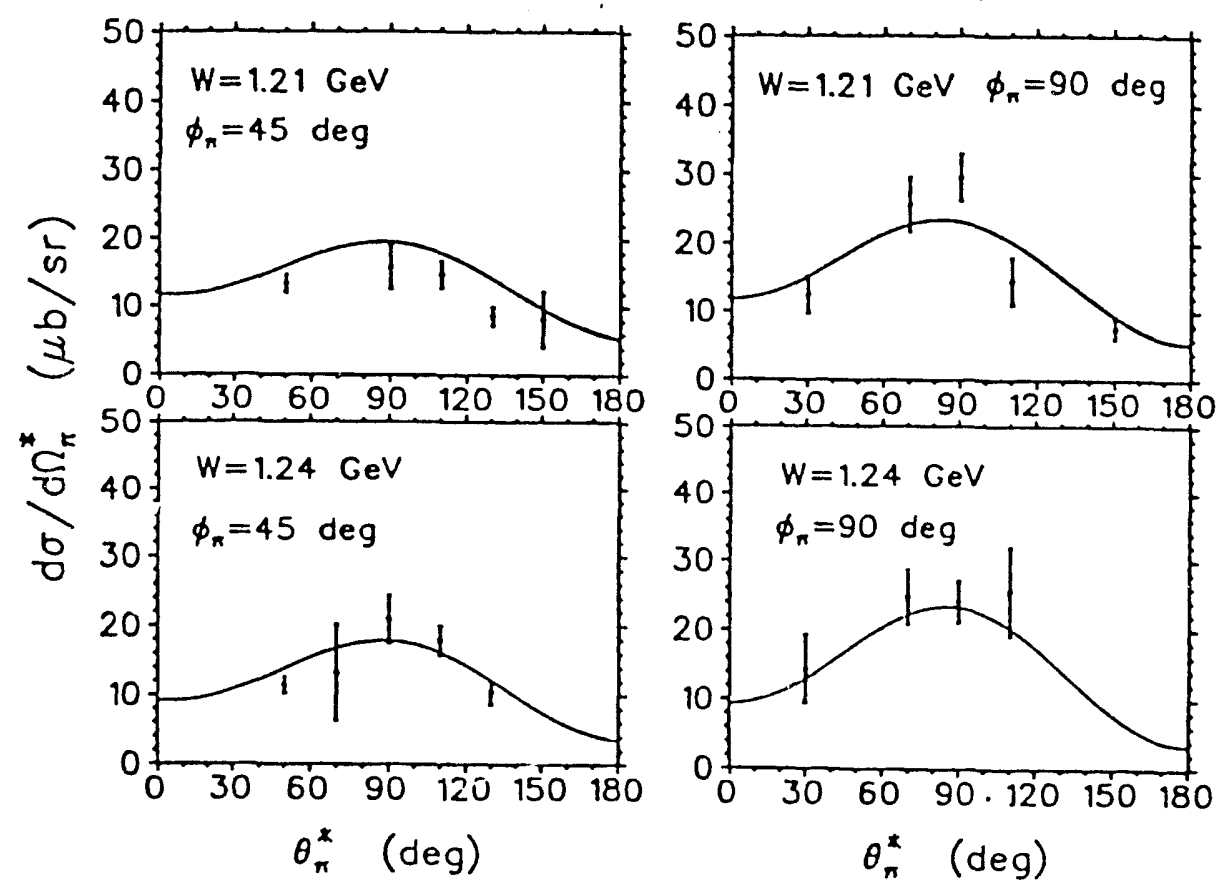

Fig. 2. Measurements and theoretical predictions for out-of-plane cross sections for the $\mathrm{p}+\overrightarrow{\mathrm{e}} \rightarrow \mathrm{p}+\mathrm{e}^{\prime}+\mathrm{X}$ reaction.

A number of other BATES experiments were also mentioned. These address topics in final state interactions, nuclear and nucleon structure, and the elastic deuteron form factors (see the plenary talk of R. Holt). Finally, a new facility for the use of internal polarized targets was described. A large acceptance toroidal spectrometer (BLAST) will permit many spin observables to be measured simultaneously for studies of nuclear and nucleon structure.

T.-S. H. Lee of Argonne described a unitary, gauge invariant model to describe the $\gamma \mathrm{N} \rightarrow \pi \mathrm{N}$ and $\mathrm{N}\left(\mathrm{e}, \mathrm{e}^{\prime} \mathrm{p}\right) \pi$ reactions starting from an effective Lagrangian. The goal is to learn about the structure of $\Delta$ and $N^{*}$ resonances. Predictions for many spin observables were made and these agree well with the extensive $\gamma \mathrm{N} \rightarrow \pi \mathrm{N}$ data in the $\Delta$ - excitation region. An example of the predictions for the ${ }^{1} \mathrm{H}\left(\mathrm{e}, \mathrm{e}^{\prime} \mathrm{p}\right) \pi$ reaction with out-of-plane kinematics is shown in Fig. 2. The need for high statistics mea- 
surements for this reaction was stressed, in order to more accurately determine the $\Delta$ and $\mathrm{N}^{*}$ form factors.

$R$. Jaffee of MIT described the nine quark structure functions probed in hard scattering inclusive processes on nucleons. Two of these functions, $f_{1}(x)$ and $g_{1}(x)$, have been measured for the proton in deep inelastic lepton scattering experiments, and $\mathrm{f}_{1}$ for the neutron has also been measured. The spin dependent function $\mathrm{h}_{1}(\mathrm{x})$ is leading twist, in addition to $\mathrm{f}_{1}$ and $\mathrm{g}_{1}$, and can be measured in the Drell-Yan process with transversely polarized beam and target. The twist- 3 functions gT and $h_{L}$ are probes of quark-gluon polarization effects, and these functions dominate in certain kinematical regions. The remaining functions e (twist-3), and $\mathrm{g}_{3}, \mathrm{~h}_{3}, \mathrm{f}_{4}$ (twist-4), mix with leading-twist functions, and are expected to be difficult to isolate. The f and $\mathrm{g}$ functions are chiral-even and can be measured with lepton scattering experiments, whereas the $e$ and $h$ functions are chiral-odd and must be studied with other processes, such as Drell-Yan.

D. Crabb of the University of Virginia described the status of CERN experiment NA47 (SMC), a measurement of $g_{1}(x)$ for the neutron using deep inelastic polarized muon scattering from a polarized deuterium target. Upgrades to the old EMC target and spectrometer have been made and a new muon polarimeter was constructed. The goal will be to test the Bjorken sum rule involving the integrals of $g_{1}$ for the neutron and proton.

M. Burkardt of SLAC described calculations of $s$ and $\bar{s}$ quark distributions in a proton. A small fraction of $s \bar{s}$ pairs live long enough for the $s$ and $\bar{s}$ quarks to interact with other partons. Differences in interaction cross sections causes the s quark distribution to be at larger $x$ than the $\bar{s}$ distribution. In the Gross-Neveu model, including spin effects, s $\downarrow$ quarks are at larger $x$ than $s \uparrow$ quarks and $\bar{s} \uparrow$ at larger $x$ than $\bar{s} \downarrow$. Since lepton scattering is only sensitive to the $s+\bar{s}$ distribution, some other process will be required to look at $\mathbf{S}$ and $\overline{\mathrm{s}}$ separately in order to test these predictions.

\section{HADRON SCATTERING}

After many years of experiments at the meson factories, the pp or isospin-1 $(I=1)$ nucleon-nucleon elastic scattering amplitudes are now reasonably well determined (to $\leqslant 5 \%$ of the largest amplitude) for energies up to $1 \mathrm{GeV}$. Much of the recent research is directed to the study of the $\mathrm{I}=0$ amplitudes, which are derived from $n p$ scattering data.

M. McNaughton of LAMPF described measurements of spin transfer parameters for $\vec{n} p \rightarrow n \vec{p}$ at $800 \mathrm{MeV}$ over a wide range of angles; see Fig. 3. This ongoing experiment was made possible by the recent commissioning at LAMPF of the new high-intensity polarized ion source, OPPIS. The data will contribute substantially to the goal of determining the $\mathrm{I}=0$ amplitudes to better than $\pm 5 \%$. In addition, the same apparatus was used to measure $K_{L L}$ for the $\vec{p} d \rightarrow \vec{n} p p$ reaction, which produces the LAMPF polarized neutron beam. The res:its suggest that this beam is about $15 \%$ more polarized at $800 \mathrm{MeV}$ than believed previously.

N. Davison of the University of Manitoba described an experiment recently completed at TRIUMF to measure spin observables for the $\vec{n} p \rightarrow p p \pi^{-}$reaction using a set of wire chambers and a scintillation counter hodoscope. This reaction is sensitive to parts of the $I=1$ inelastic cross section other than the strong $N \Delta^{+}$ channel, as well as to $I=0$ pion production. Elastic scattering and $\mathrm{np} \rightarrow \mathrm{pp} \pi^{-}$events have been clearly identified, and a density-matrix analysis of the data is planned. 
Similar experiments at higher energies are being performed at Saturne and approved for LAMPF.

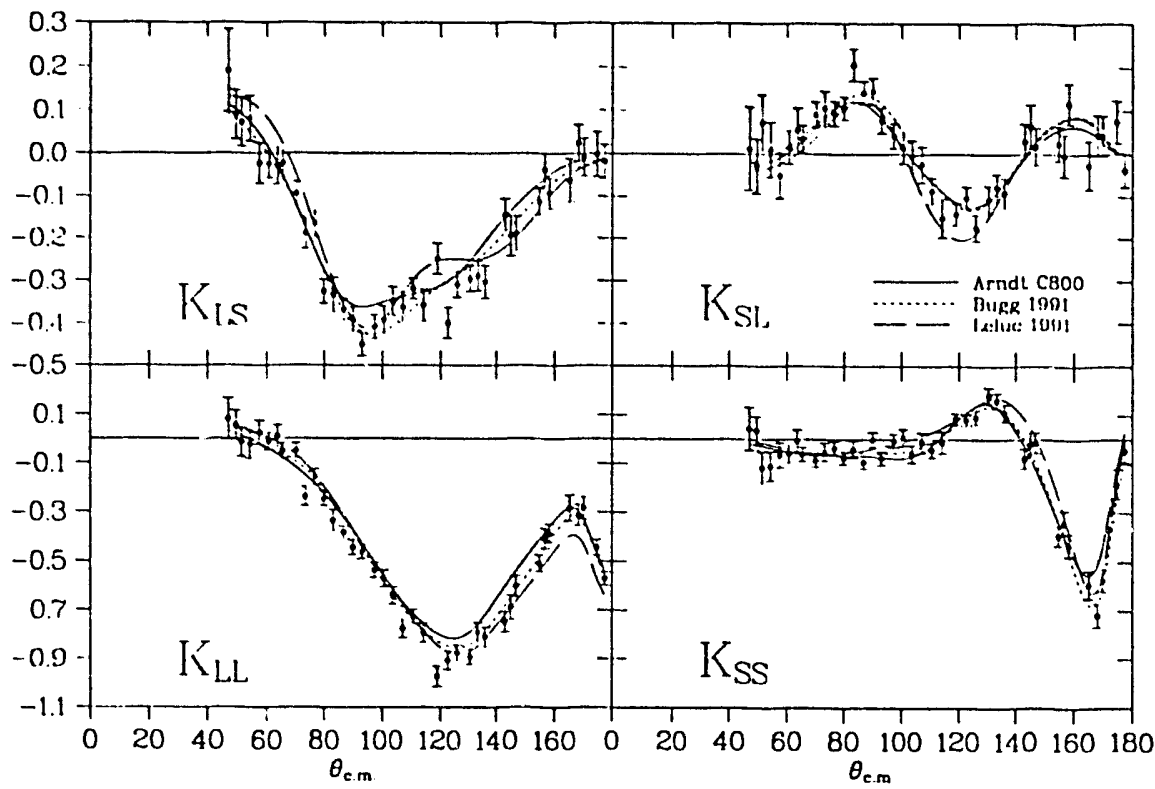

Fig. 3. Data for $\vec{n} p \rightarrow n \vec{p}$ spin observables at $T_{n}-=800 \mathrm{MeV}$ as a function of c.m. angle with various phase shift predictions.

New data were presented by M. Beddo of Argonne for $\Delta \sigma_{L}(n p)$, the total cross section difference between antiparallel and parallel spin states for longitudinally polarized neutron beam and proton carget. Sizeable energy dependent structure is observed for $\Delta \sigma_{L}(n p)$ and $\Delta \sigma_{L}(I=0)=2 \Delta \sigma_{L}(n p)-\Delta \sigma_{L}(p p)$, using data from PSI, Saturne, and LAMPF as shown in Fig. 4. Good agreement is found at overlappir, energies for the different data sets. These results do not agree well with current phase shift solutions.

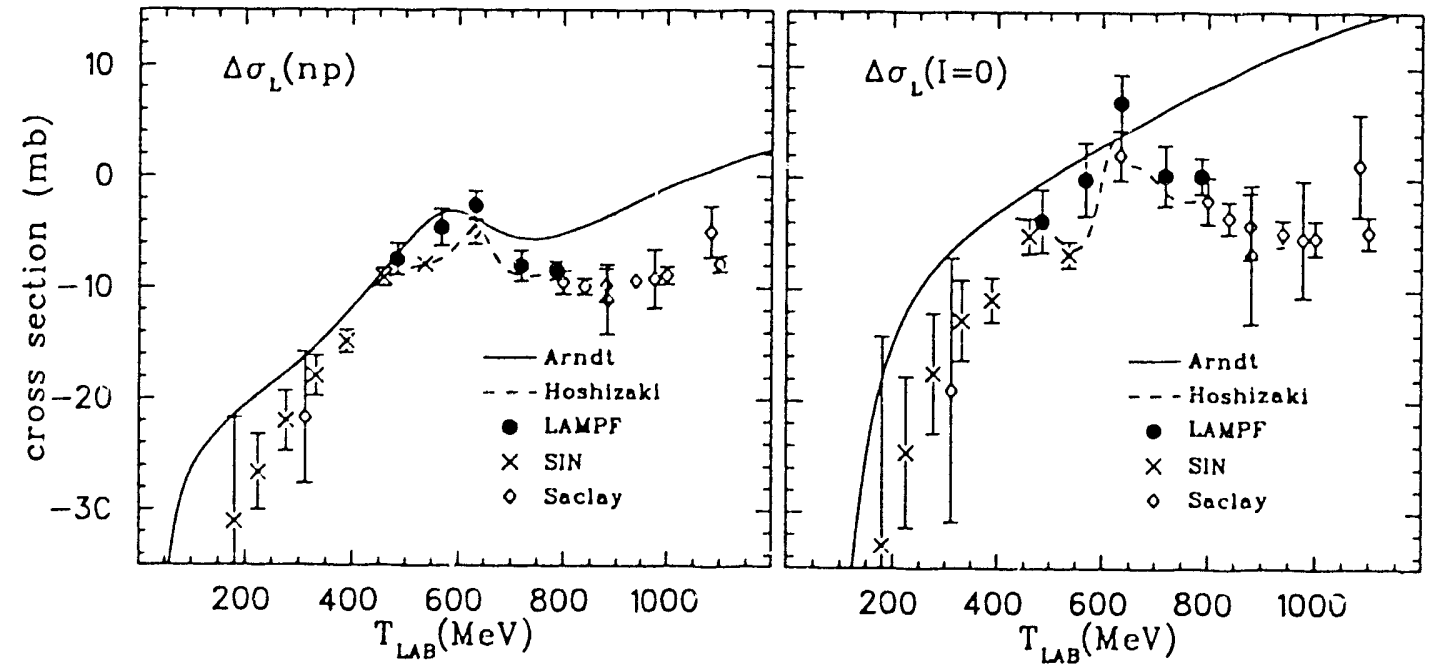

Fig. 4. Measurements of $\Delta \sigma_{L}(n p)$ and extracted results for $\Delta \sigma_{L}(I=0)$ from various laboratories. The solid curve is a phase shift production of $\mathrm{R}$. Arndt. 
The structure in $\Delta \sigma_{L}(I=0)$ was discussed in more detail by $H$. Noya of Hosei Universily. Japan. A diquark-cluster model of dibaryons was discussed and the resulting dibaryon mass spectrum was presented. Predictions of other structure in $\Delta \sigma L(I=0)$ in this energy range were showil. It was noted that inelastic thresholds for $\mathrm{I}=0$ interactions occur at a considerably higher energy than the observed structure in $\Delta \sigma_{L}(I=0)$ and than the $N \Delta$ threshold in the $I=1$ channel.

I. Strakovsky of LNPI Gatchina, Soviet Union and of TRIUMF discussed results from his phase shift analysis (PSA) for the $\mathrm{I}=1 \mathrm{pp} \Leftrightarrow \pi \mathrm{d}$ reaction between $\mathrm{T}_{\mathrm{p}}=$ $440-1236 \mathrm{MeV}$ or $\mathrm{T}_{\pi}=76-474 \mathrm{MeV}$. Combining his results with those from the VPI PSA for $N N \rightarrow N N$ and from TRIUMF for $\pi d \rightarrow \pi d$, it was observed that a looping in the $2^{+}$and $3^{-}$wave is seen near the same total energy in all three analyses. Sizeable changes were shown to occur in the total cross section difference data, $\Delta \sigma \mathrm{T}$ and $\Delta \sigma \mathrm{L}$, for the reactions $\mathrm{pp} \rightarrow \pi \mathrm{d}$ and $\mathrm{pp} \rightarrow \mathrm{pN} \pi$ as a function of energy. Measurements of $\Delta \sigma_{\mathrm{L}}^{\mathrm{d}}=\int \mathrm{T}_{20}(\mathrm{~d} \sigma / \mathrm{d} \Omega) \mathrm{d} \Omega$ and of $\Delta \sigma \sigma_{T} \mathrm{~d}=\int \mathrm{T}_{22}(\mathrm{~d} \sigma / \mathrm{d} \Omega) \mathrm{d} \Omega$ for $\pi \mathrm{d} \rightarrow$ pp were strongly encouraged, as well as a coupled channel analysis of $\mathrm{NN}, \pi \mathrm{d}$, and resonant and nonresonant $N N \pi$ in order to properly address the question of the possible existence of broad $\mathrm{I}=1$ dibaryons.

At higher energies, R. Phelps of the University of Michigan described pp elastic analyzing power (A) measurements at the Brookhaven AGS and UNK. Final results at $24 \mathrm{GeV} / \mathrm{c}$ and $\mathrm{p}_{\perp}{ }^{2}=3-7 \mathrm{GeV} 2 / \mathrm{c}^{2}$ were presented from the Brookhaven polarized beam experiments. The data show that $\mathrm{A}$ increases sharply with increasing $\mathrm{p}_{\perp}{ }^{2}$. Plans for measurements at $400 \mathrm{GeV}$ (INK) with an internal, ultra cold polarized gas jet target of atomic hydrogen were presented. It is hoped to measure A for pp elastic scattering out to $\mathrm{p}_{\perp}^{2}=8 \mathrm{GeV}^{2} / \mathrm{c}^{2}$ and for inclusive production of $\mathrm{p}$ 's, $\pi$ 's, and $\mathrm{K}$ 's beginning in 1993. The hardware will include "Roman pots" to detect the forward protons and a magnetic spectrometer instrumented with wire chambers for the recoil proton and inclusively proriuced particles.

D. Sivers discussed spin predictions for two exclusive reactions, $p p \rightarrow p p$ and $\gamma d \rightarrow n p$, in the quark helicity conservation limit. Both processes obey constituent counting rules for cross sections at relatively low energies. The nucleon-nucleon amplitudes were modeled using quark interchange and the Landshoff mechanism. Predictions for A and ANN were compared to the Michigan pp $\rightarrow$ pp data from the ZGS and AGS, suggesting that these calculations may apply at $12 \mathrm{GeV} / \mathrm{c}$ or below. The reaction $\gamma d \mathrm{~d} \rightarrow \mathrm{np}$ was studied in the constituent interchange model. In the limit where quark helicity is conserved, nine of the twelve amplitudes for this process vanish dynamically. Predictions of spin observables $(\vec{\gamma} d \rightarrow n p, \overrightarrow{\gamma d} \rightarrow n \vec{p}, \gamma \vec{d} \rightarrow n \vec{p})$ were presented. It would be interesting to compare these predictions with measurements at a number of energies to determine where the changeover between nuclear and quark physics occurs in this reaction.

A remarkable wealth of new results from the Fermilab $200 \mathrm{GeV}$ polarized beam program was presented by N. Akchurin of the University of Iowa, R. Skeen of Rice University and A. Yokosawa of Argonne. An improved measurement of the beam polarization, confirming beam design expectations, was reported. The polarimeter was based on the Coulomb-nuclear interference process in small angle pp and $\bar{p}$ p elastic scattering. The measurement demonstrates the practicality of this type polarimeter for higher energy polarized beams as well.

A variety of new inclusive production asymmetries were shown. The magnitude of the single spin asymmetries for $A=A_{N}$ for $\vec{p} p \rightarrow \pi^{ \pm} X, \vec{p} p \rightarrow \pi^{\circ} X$ and 


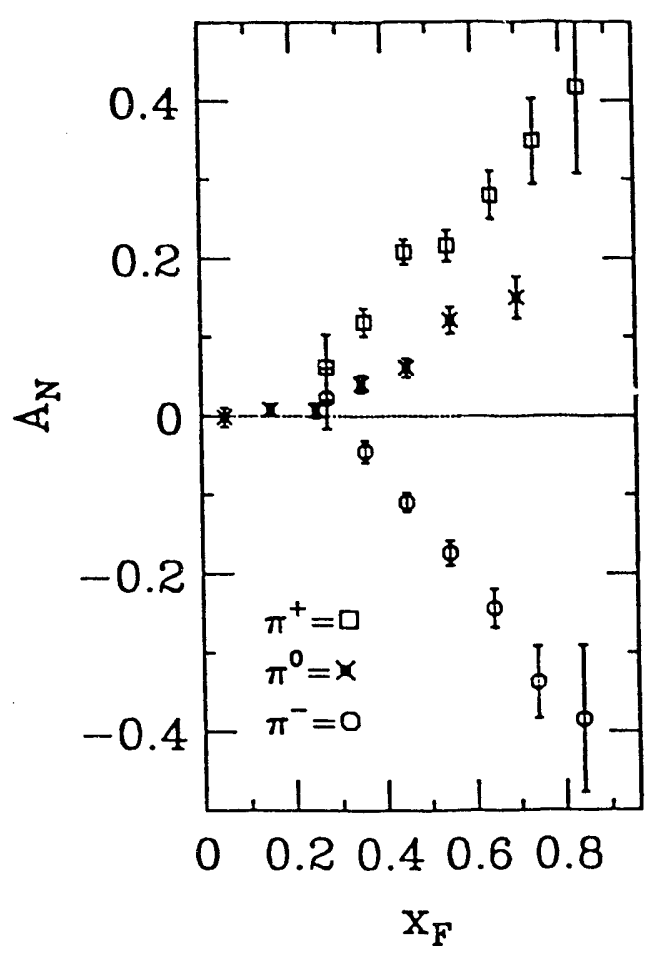

$\overrightarrow{\overline{\mathrm{p}}} \mathrm{p} \rightarrow \pi^{\circ} \mathrm{XX}$ all increase to sizeable values with increasing $\mathrm{XF}$ for $0.7<\mathrm{pT}$ $<2.0 \mathrm{GeV} / \mathrm{c}$; see Fig. 5. In addition, AN increased to values $\sim 0.4$ for $\mathrm{PT}_{\mathrm{T}} \gtrsim$ $3.5 \mathrm{GeV} / \mathrm{c}$ and $\mathrm{xF} \simeq 0$ in $\overrightarrow{\mathrm{p} p} \rightarrow \pi^{-} \mathrm{X}$. Data for $\overline{\overline{\mathrm{p}}} \mathrm{p} \rightarrow \pi^{\circ} \overline{\mathrm{X}}$ and $\overline{\mathrm{p}} \mathrm{p} \rightarrow \eta \mathrm{X}$ exhibited similar behavior, although the statistical errors were large. Finally, data for $A_{N}(\vec{p} p \rightarrow \Lambda X)$ and $A_{L L}$ $\left(\vec{p} \vec{p} \rightarrow \pi^{\circ} X\right)$ were presented. These many new results will hopefully provide ample clues to understand the origin of the large spin effects observed at this high energy.

Fig. 5.

Measurements of asymmetry in inclusive production of pions at 200 $\mathrm{GeV} / \mathrm{c}$ by polarized protons as a function of $x_{F}$.

Going to still higher energies, G. Bunce of Brookhaven discussed the possibility of collisions of $250 \mathrm{GeV}$ polarized protons on $250 \mathrm{GeV}$ polarized protons at RHIC. The need for a number of "snakes" on the AGS and RHIC was explained. The expected luminosity $\mathcal{L} \sim 10^{32} / \mathrm{cm}^{2} / \mathrm{sec}$ would permit adequate rates to perform asymmetry measurements up to $\mathrm{PT} \sim 20 \mathrm{GeV} / \mathrm{c}$ for direct- $\gamma$ production, and up to $\sim 50$ $\mathrm{GeV}$ for jet production in a one month run. Additional details of the types of physics that could be explored are given in the plenary talk of J. Collins.

\section{SPIN TECHNOLOGIES}

The status of polarized electron and proton sources, solid polarized targets, and Siberian snakes to eliminate the effects of depolarizing resonances were discussed in five talks. All four areas showed considerable progress in the past few years.

L. Cardman of the University of Illinois reviewed polarized electron source technology. Gallium arsenide ( $\mathrm{GaAs}$ ) sources have found considerable applications in the past with polarizations $\sim 40 \%$, quantum efficiency $\sim 5 \%$, long lifetime and high peak currents. Recently a number of "custom crystals" have been made which have demonstrated polarization of 70-85\%. Some of these crystals are composed of alternative layers of two materials with different lattice spacing, thereby inducing strains in the crystal. These strains remove the energy degeneracy that limit the maximum theoretical polarization to $50 \%$ for GaAs. The quantum efficiency of the new crystals is presently low, but rapid improvements are being made, and practical applications in the near future are foreseen.

O. van Dyck of LAMPF presented an overview of the progress on optically pumped polarized ion sources (OPPIS) at LAMPF, KEK, TRIUMF, and the Moscow 
Meson Factory. These sources transfer spin angular momenta from high power lasers to $\mathrm{H}^{-}$beams through a charge exchange process in an alkali vapor. Typical maximum $\mathrm{H}^{-}$polarizations of $65-70 \%$ and d.c. currents of $20 \mu \mathrm{A}$ or peak currents of $100 \mu \mathrm{A}$ have been achieved, which represent a very sizeable improvement over the Lamb-shift polarized source used previously at LAMPF. Studies of the OPPIS performance were described that may boost the present intensities by as much as another order of magnitude. Interlaboratory cooperation has been very important in the development of OPPIS technology.

D. Crabb of the University of Virginia discussed the high proton polarization $(\sim 96 \%)$ observed in irradiated $\mathrm{NH}_{3}$ when cooled to $1 \mathrm{~K}$ in a $5 \mathrm{~T}$ field. This material shows a rapid buildup time, and it can be polarized with a ${ }^{4} \mathrm{He}$ refrigerator instead of the more complex ${ }^{3} \mathrm{He}$ or dilution refrigerators. Irradiated ND3 has been tested at low temperatures up to $3.5 \mathrm{~T}$, giving deuteron polarizations of $30 \%$, but the buildup time was measured in hours. Measurements with ND3 at $1 \mathrm{~K}$ and $5 \mathrm{~T}$ would be quite interesting for experiments needing polarized "neutron" targets. O. Hausser and J. Comfort also described polarized nuclear targets, including ${ }^{6,7} \overrightarrow{\mathrm{Li}},{ }^{13} \overrightarrow{\mathrm{C}},{ }^{15} \overrightarrow{\mathrm{N}}$ and optically pumped ${ }^{3} \mathrm{He}$ gas, in other sessions at this conference.

T. Roser of the University of Michigan and Brookhaven presented the results of an experimental test of the "Siberian Snake" concept. Magnets are used to rotate the beam spin each turn, without affecting the orbit, in order to cancel the effects of depolarizing resonances in circular accelerators. The test was performed at the Indiana University cooler ring with a solenoid for the snake. The experimetnal data confirmed the expected behavior for both an isolated imperfection and an isolated intrinsic depolarizing resonance in the cooler ring.

Application of the snake concept to higher energy accelerators was discussed by both T. Roser and L. Ratner of Brookhaven. Details were presented of a proposed test of a partial snake at the AGS. The snake would consist of an air core solenoid of $90 "$ effective length. This test would be cruicial to look for the compounded effects of small depolarization losses at each of many resonances, which could make the snake concept impractical above some energy.

\section{CONCLUSIONS}

Spin physics at this conference was shown to be producing exciting results on a wide variety of topics, from very low to very high energies. Symmetry tests continue to improve limits, while searching for new physics. Lepton scattering experiments are important to improve the understanding of the nucleon spin structure, as well as other physics, so that over half of the future BATES and CEBAF programs are expected to involve spin. Impressive progress has been made in understanding nucleon-nucleon interactions at intermediate energies. At high energies, strong interaction spin effects persist and grow with $\mathrm{PT}$ and $\mathrm{XF}$. Finally, there is rapid progress in polarized source and target technology, and in the use of snakes to overcome depolarizing resonances in circular proton accelerators. It will be interesting to see what new physics will be learned with spin at LEP and perhaps RHIC in the future.

$\dagger \quad$ Work supported in part by the U.S. Department of Energy, Division of High Energy Physics, Contract W-31-109-ENG-38. 


\section{DISCLAIMER}

This report was prepared as an account of work sponsored by an agency of the United States Government. Neither the United States Government nor any agency thereof, nor any of their employees, makes any warranty, express or implied, or assumes any legal liability or responsibility for the accuracy, completeness, or usefulness of any information, apparatus, product, or process disclosed, or represents that its use would not infringe privately owned rights. Reference herein to any specific commercial product, process, or service by trade name, trademark, manufacturer, or otherwise does not necessarily constitute or imply its endorsement, recommendation, or favoring by the United States Government or any agency thereof. The views and opinions of authors expressed herein do not necessarily state or reflect those of the United States Government or any agency thereof. 

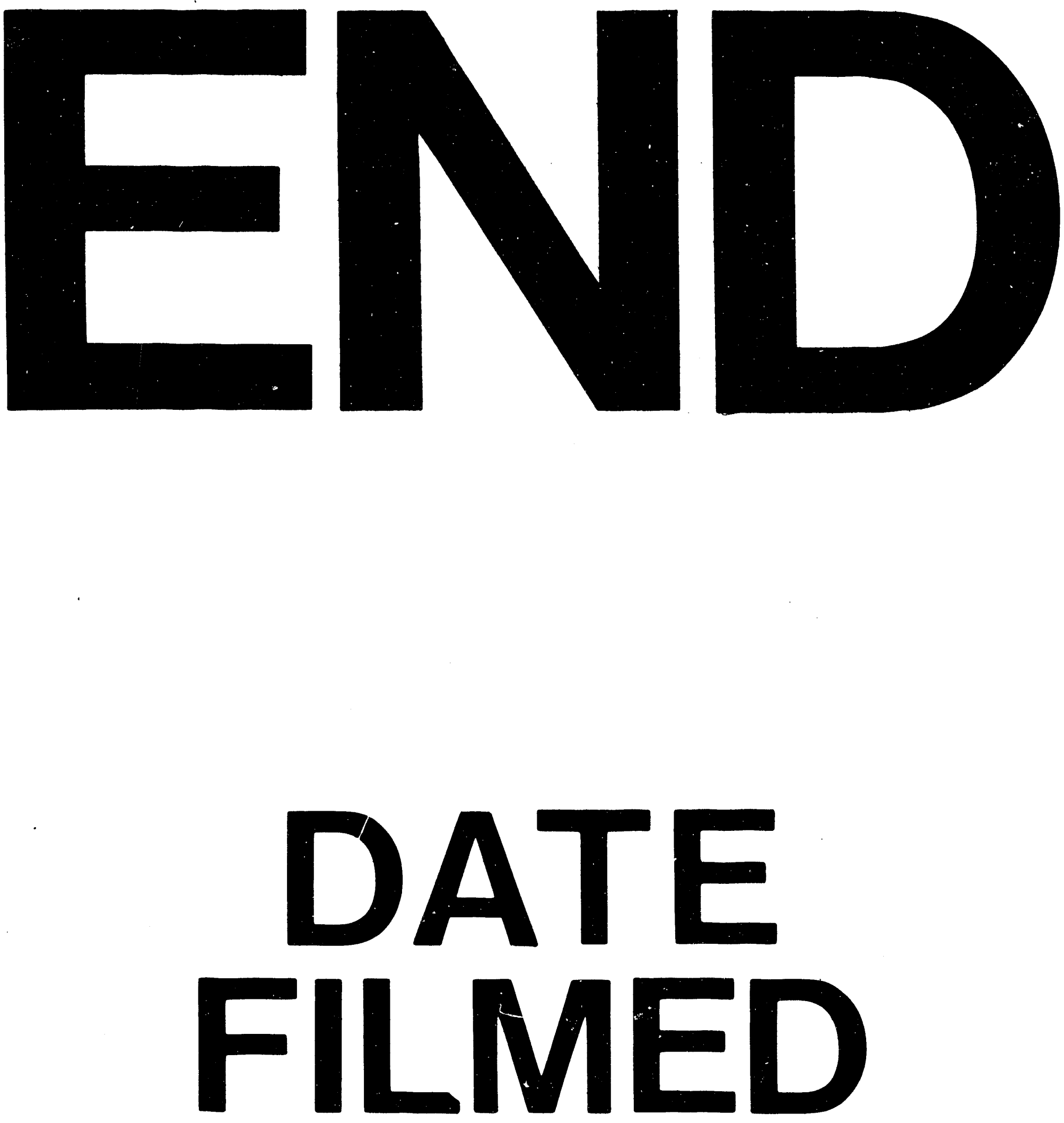

1

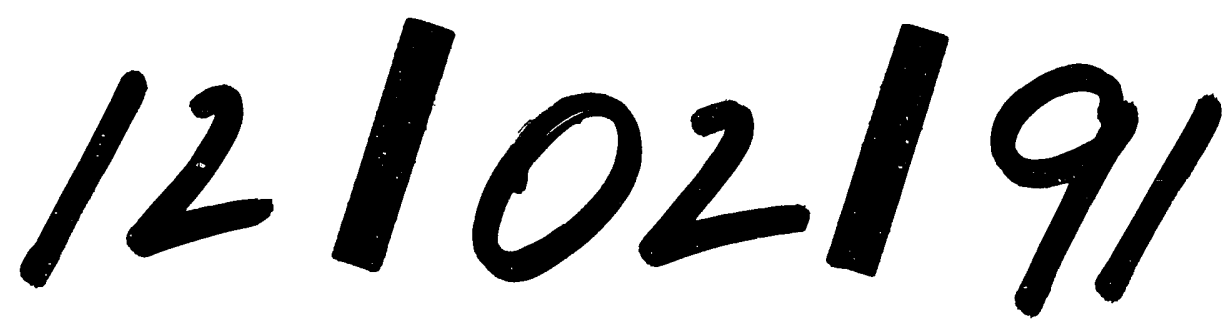

$\bar{Z}$ 
